Archived version from NCDOCKS Institutional Repository http://libres.uncg.edu/ir/asu/

\title{
Appalachỉan
}

B O O N E, N O R T H C A R O L I N A

\section{Stage Transition Duration In Patients Poststroke}

\author{
Authors: \\ Youngsun Kim \& Gary H. McCullough
}

\begin{abstract}
The purpose of this study was to investigate the relationship between prolonged stage transition duration

(STD) and aspiration. STDs and aspiration ratings were made from videofluoroscopic examinations previously obtained for other studies. Three groups of subjects were examined: (1) 20 stroke patients who aspirated, (2) 31 stroke patients who did not aspirate, and (3) 15 normal subjects. Aspirators poststroke had the longest STD of the three groups. Furthermore, results indicated that STD correctly predicted the presence of aspiration $75 \%$ of the time and correctly predicted the absence of aspiration in stroke patients 93\% of the time. Nonaspirating stroke participants had similar patterns to the normal subjects.

Implications for these findings are discussed.
\end{abstract}

Youngsun Kim \& Gary H. McCullough (2007) "Stage Transition Duration In Patients Poststroke" Dysphagia \#22 pp.299-305 Version of Record Available At www.springer.com [DOI: 10.1007/s00455-007-9085-4] 


\section{$\underline{\text { Introduction }}$}

The primary purpose of swallowing is to safely transport food from the mouth to the stomach. Safe and proper ingestion of food and liquid is critical to survival. While nutritional status can often be maintained by nonoral means, quality of life may be tragically diminished by such mechanical alimentation.

Swallowing can be divided into three physiologic stages: oral, pharyngeal, and esophageal [1]. As a bolus is propelled from the oral cavity into the pharyngeal cavity, the pharyngeal stage of the swallow should be triggered. Triggering of the pharyngeal swallow means that the following physiologic events should occur: (1) velopharyngeal apposition to the posterior pharyngeal wall to seal the nasopharynx and prevent food or liquid from entering the nasopharynx; (2) elevation and anterior movement of hyoid bone and thyroid cartilage which contribute to epiglottic closure and the opening of the upper esophageal sphincter (UES); (3) the closure of true and false vocal folds and anterior tilt of the arytenoids to protect the airway from penetration or aspiration of the bolus; and (4) opening of the cricopharyngeal sphincter to allow passage of the bolus into the esophagus [2]. Meanwhile, the bolus is propelled downward by initial oral and tongue base force in conjunction with pharyngeal constrictors.

The bolus passage from the oropharynx into the hyopharynx can be referred to as "the stage transition" [3]. This is not to suggest that it is the only transition that occurs between stages of swallowing; the transition between the pharyngeal stage and the esophageal stage is important and time-dependent as well. Still, in this case the stage transition refers specifically to the transition of the oral stage into the pharyngeal stage. The end of the oral stage has been defined radiographically in this study as the time when any barium passes the ramus of the mandible [3]. It has been reported previously that this is the area that most closely correlates with the anatomical point of reference targeted, the anterior faucial pillars [1]. The initiation of the pharyngeal phase, as defined in association with STD [3], is defined as the time when the hyoid begins its anterior excursion. Thus, stage transition duration (STD) is the time from first barium passing the ramus of the mandible until the beginning of maximum anterior hyoid excursion. Any lag in time between the bolus passing the ramus of the mandible and the initiation of anterior hyoid excursion then can be referred to as "stage transition 
duration." If the stage transition is zero seconds, then hyoid excursion began simultaneously with the bolus entering the pharynx. If the stage transition is a negative number, this means hyoid excursion began before the bolus ever reached the pharynx. Robbins et al. [3] reported that in normal, young adults, STDs of 0 or negative were the norm. In other words, the initiation of hyoid excursion began before or when the bolus passed the ramus of the mandible. For older participants, however, this was not the case. Rather, hyoid excursion began after the bolus had entered the pharynx, resulting in a positive number for STD. The participants in the Robbins et al. study were tested with 2-ml boluses, limiting comparisons of results. Still, STD for older normals was greater than 0 and approached 0.3-0.4 s, similar to our data [5]. The delay in hyoid excursion leaves the airway unprotected for a prolonged period of time, which increases the risk of aspiration; but it must be clarified that aspiration did not typically occur as a result of normal aging processes which slowed the stage transition.

Many stroke patients with dysphagia also exhibit prolonged transitions between the oral and pharyngeal stages. Robbins and Levine [4] reported that those who suffered a right cerebral vascular accident (CVA) have been observed to have more problems with the timing of pharyngeal stage components and subsequent penetration/aspiration than those with a left CVA. Patients with other types of cerebrovascular disease such as lateral medullary syndrome may also exhibit severe delays in the triggering of pharyngeal swallow. With bilateral damage they may have no swallow response at all. Other patients at risk for delays in transition between the oral and pharyngeal stages include those with disorders such as traumatic brain injury (TBI), Parkinson's disease, multiple sclerosis, bulbar palsy, and head and neck cancer [1]. Adult dysphagic patients with these problems showed a prolonged transition between the end of the oral stage and the beginning of the pharyngeal stage, or the initiation of the pharyngeal swallow, which have been linked to aspiration. In addition, the presence of a delayed initiation of the pharyngeal stage may be linked to negative health outcomes from aspiration. At this point it seems prudent to emphasize that aspiration can occur for many reasons, of which prolonged STD is only one. Reduced hyolaryngeal excursion, weak tongue-base propulsion, weak pharyngeal wall propulsion, or early, late, or reduced opening of the UES can all lead to aspiration. However, thin-liquid aspiration before or during the swallow does result in aspiration in a high percentage of stroke patients; this is the focus of this article.
While normative data have been collected for STD in normal populations [3, 5], to our knowledge no studies have compared stroke patients with and without aspiration. The purpose of this study was to evaluate the oropharyngeal stage transition duration in three groups of subjects. The three groups were as follows: (1) 20 stroke patients who aspirated (aspirators), (2) 31 stroke patients who did not aspirate (nonaspirators), and (3) 15 normal subjects within the appropriate age range for stroke patients. Normal subjects were between 60 and 80 years of age (mean $=76.93$ ), which was the age range for $90 \%$ of the sample of stroke patients who aspirated (mean $=69.80)$ and who did not aspirate (65.64).

Because X-ray swallow studies are necessarily short, best and worst case scenarios for oral ingestion are not always visible. We sit them upright, feed them by spoon or cup, and ask them to swallow-several times if necessary. Thus, it is entirely probable that patients exist who do not aspirate in the fluoroscopy suite but do aspirate under normal circumstances, where posture is more reclined and swallowing is not attempted on verbal cue. It is, therefore, essential to collect as much information on specific physiologic aspects of the swallow as possible to determine how much of a risk there is for aspiration or other symptoms related to dysphagia. If age-based duration measures of swallowing, like STD, can help the clinician define a range in which aspiration is more or less likely to occur for specific populations such as those who suffer a stroke, then we begin to expand our knowledge base using videofluoroscopy and, thus, feel more confident in what we may not clearly see in the fluoroscopy suite. Therefore, means and ranges of STDs that lead to aspiration versus no aspiration in patients poststroke may enhance our overall evaluation and improve our treatment recommendations. Comparisons with normal subjects may help define whether differences also exist between stroke patients and normals outside the occurrence of aspiration. If nonaspirating stroke patients are similar to normals in STD, then our distinction for prolonged and normal STDs is strengthened. Then again, perhaps a graded prolongation of STD exists from normal to nonaspirating stroke to aspirating stroke. Moreover, means and ranges of STDs, if truly different for these groups, can allow us to begin to look not only at symptoms of dysphagia, such as aspiration, but also at outcomes of health status and nutrition.

Thus, quantification of STD could be an important marker that defines who is at risk for aspiration. It may also provide insight into how much prolongation is too long and when treatment or compensation should be initiated. Also, STD data 
will help the clinician to decide whom he/she should monitor more closely at bedside and followup. Again, this is not to suggest that STD is the only cause of aspiration, but it is one cause and the most likely cause for aspiration before and during the swallow for patients poststroke.

\section{Method}

\section{Subjects}

Videofluoroscopic swallowing examinations (VFSEs) were recorded on 60 stroke patients for a prior investigation [6]. These were consecutively admitted stroke patients in our hospitals, not just ones referred for possible dysphagia. In that prior study, 55 of the 60 were male, mostly in the Nashville, Tennessee, VA Medical Center. The mean age was 67.8 years and the mean number of days after onset was 5.98. Brain imaging revealed that 44 of the 60 suffered a single stroke, and 16 suffered two or more strokes. Twenty-nine has sustained a cortical stroke or strokes. Eleven of these involved the right hemisphere, 17 involved the left hemisphere, and one was bilateral. Fourteen had sustained subcortical stroke or strokes. Nine of these were on the right side, four were on the left, and one was bilateral. Six patients had sustained a brain stem stroke or strokes. Two of these were on the right side, two were on the left, and two were bilateral. Three patients had sustained a cerebellar stroke, three had a mixed localization, and the lesion in three others could not be localized. Twenty-two patients aspirated and 38 did not. For this investigation, 20 of the 22 patients with aspiration and 31 of the 38 patients with no aspiration were selected for STD measurement. Nine patients were excluded from this study because of poor visibility of the hyoid bone during the swallow. Among stroke patients in this sample, six aspirators and four nonaspirators had a history of pneumonia, and ten aspirators and eight nonaspirators suffered from poor nutrition. Seven aspirators had a feeding tube at the swallowing exam. Nonaspirators in this investigation did not show aspiration, but other signs of dysphagia existed in many, such as tongue weakness, dysphonia, wet voice quality, secretion problem, and/or poor nutrition.

All VFSEs from the prior study were reviewed and analyzed by the principal investigator. The presence or absence of aspiration was reported, as was the timing of the aspiration: before, during, or after the swallow. All 22 of the patients who aspirated did so on 5or 10-ml thin-liquid boluses (and sometimes thick liquids) either before or during the swallow. Thus, no patients in this sample aspirated after the swallow or on puree or solid consistencies. Therefore, for the purposes of this study, all thin-liquid swallows were reanalyzed to determine STD for 5 and $10 \mathrm{ml}$. No puree or solid consistencies were rated.

In addition, this study used stage transition duration (STD) data on 15 normal subjects also recorded from a prior investigation [7]. Normal subjects and stroke patients were not matched up perfectly because of the use of existing databases, but all participants fell within the same age ranges, largely between 60 and 80 years of age, based on the mean age of stroke patients in this analysis. Previous studies showed that older subjects had longer STD than younger subjects $[3,5]$, and normative values were derived specifically for individuals between the ages of 60 and 80 (see Table 1). These 15 normal subjects served as a control group for
Table 1. The mean and standard deviation (SD) of age (years) of the three subject groups

\begin{tabular}{lcccc}
\hline Groups & $N$ & Mean & SD & Range \\
\hline Aspirators & 20 & 69.80 & 8.91 & 33.00 \\
Nonaspirators & 31 & 65.64 & 9.83 & 42.00 \\
Normals & 15 & 76.93 & 4.59 & 14.00 \\
\hline
\end{tabular}

comparison with stroke patients with and without aspiration Normal subjects were screened for neurologic or structural abnormalities that would interfere with swallowing by a comprehensive questionnaire and oral motor examination. There was no attempt for normal participants to screen out diabetes, HTN, arthritis, or other health issues because it was deemed necessary during the normative data collection to examine true "normals" and not be so selective that data were only applicable to a handful of "supernormals." Complete medical histories, therefore, would be too lengthy to detail for each normal participant. In general, however, they were individuals who met screening criteria, appeared healthy, ambulated into the clinic, and were able to participate with full mental and physical faculties. No reports of any difficulties with nutritional intake were received.

\section{Videofluoroscopic Swallowing Examination (VFSE)}

Videofluoroscopic swallowing examination (VFSE) data were collected on stroke patients [6] and normals [7] using the same research protocol. The fluoroscopic tube was focused in the lateral plane on the oral cavity (the lips anteriorly to the pharyngeal wall posteriorly), and the nasopharynx (superiorly) to just below the UES area (inferiorly). Each subject swallowed a wide array of bolus sizes and consistencies, including sizes from 5-ml to 3 ounces and consistencies from thin liquid to solid. For this investigation, however, data were analyzed for only two $5-\mathrm{ml}$ boluses and two $10-\mathrm{ml}$ boluses of thin liquid. The boluses were a mixture of water and barium sulfate powder $(50 / 50$ water and E-Z M barium sulfate powder for suspension) [6,7]. VFSE began with two swallows of each thin liquid, 5 and $10 \mathrm{ml}$. Each subject was instructed by the clinician to swallow after putting the liquid in his/her mouth by pill cup. The only variability occurred due to "bailout criteria." Though these criteria were identical for both normal and stroke participants, bailout criteria were used only if aspiration appeared to place them at risk and were, thus, used for only some of the stroke participants. All other aspects of VFSEs were identical for normals and stroke patients.

\section{Procedures for Temporal Measurement}

Dysphagia manifests itself as a disruption in one of two primary types of physiologic impairment (and typically both): (1) problems with the timing, distance, or strength of movement of oropharyngeal structures; and/or (2) misdirection of bolus flow is a result of physiologic dysfunction. The former is typically the cause of the latter. In other words, decreased hyoid excursion or poorly timed initiation of hyoid excursion can result in aspiration of liquid before or during the swallow. Data regarding the occurrence of aspiration for subjects in this investigation were already recorded and analyzed to place participants into one of the three groups. Aspiration was recorded simply as present or absent.

This study focused its analyses on one aspect of bolus flow (movement of the bolus from the oral cavity into the pharynx) and 
one aspect of structural movement (initiation of hyoid excursion). To accurately analyze the temporal sequence of events, slow-motion, frame-by-frame analyses were performed using a 100-ms videotimer. Thus, for each swallow evaluated, the time (in milliseconds) that the bolus reached the ramus of the mandible and the time that the hyoid began its anterior excursion were recorded. The measure of STD can then be described as the relationship between the following reference points: (1) bolus flow into the pharynx and (2) initiation of maximum hyoid excursion. For each subject a worksheet was used to record these times (in milliseconds) for each point of occurrence. Then, the STD was computed using the formula: STD = (initiation of maximum hyoid elevation) - (first sign of bolus in the pharynx). STD values were then compared across the three groups of participants: aspirating stroke patients, nonaspirating stroke patients, and normal participants. Thin liquids were used because they are the primary concern for individuals with delayed transitions between the oral and pharyngeal stages. Aspiration was defined as the entry of the liquid below the true vocal folds [1].

\section{Statistical Analysis}

Each group mean, standard deviation (SD), and range of 5-ml and 10-ml thin-liquid boluses were generated by averaging each subject's two swallows in each thin liquid. A discriminant analysis was completed for STD. Both STD data of $5 \mathrm{ml}$ and $10 \mathrm{ml}$ were submitted for the discriminant function analysis as independent variables and three groups were submitted as dependent variables. Because this study had three groups, two discriminant functions were performed. The first discriminant function separated aspirators from nonaspirators and normal subjects. The second discriminant function compared only two groups, nonaspirators and normal subjects. Wilkin's lambda was used to determine the significance of each function, and the criterion of the significance level was set at $p<0.05$. Discriminant functions were used to predict classification membership of each group. The classification results showed both the percentage of correctly classified subjects and those that were misclassified.

\section{Results}

\section{Reliability}

For interjudge reliability, a second independent judge analyzed the designated swallows of 13 randomly selected subjects (52 swallows, 20\%). The measurements of the principal investigator and second judge were compared using a Pearson's correlation coefficient. A significant correlation was observed ( $r=0.86, p<0.01)$. For intrajudge reliability, the principal investigator reanalyzed the same 13 subjects a second time. Intrajudge reliability was also significant $(r=0.92, p<0.01)$.

\section{Stage Transition Duration (STD)}

Table 2 provides the mean duration, the standard deviation, and range for the three subject groups on
STD. For the aspirators, the initiation of maximum

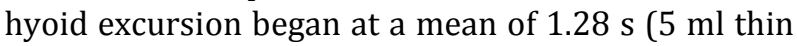
liquid) and $1.12 \mathrm{~s}$ (10 $\mathrm{ml}$ thin liquid) after the bolus reached the ramus of the mandible. For the nonaspirating stroke patients, initiation of maximum hyoid excursion occurred at a mean of $0.40 \mathrm{~s}(5 \mathrm{ml}$ thin liquid) and $0.36 \mathrm{~s}$ (10 ml thin liquid) after the bolus reached the ramus of the mandible. Finally, for normal, age-matched participants, initiation of maximum hyoid excursion began at a mean of $0.19 \mathrm{~s}(5 \mathrm{ml}$ thin liquid) and $0.23 \mathrm{~s}$ (10 $\mathrm{ml}$ thin liquid) after the bolus reached the ramus. Thus, the mean STD for each group was progressively shorter (aspirators to nonaspirators to normal). While STD was shorter for $10-\mathrm{ml}$ boluses in the two groups of stroke patients, STD was longer for $10-\mathrm{ml}$ boluses in the normals.

Figure 1 shows a boxplot for the three subject groups on STD. The stroke patients who aspirated clearly showed longer STDs than the nonaspirating stroke patients and the normal subjects. The standard deviation and range for aspirating stroke patients is also much greater than for nonaspirating stroke patients and normals. One of the nonaspirating stroke patients (No. 13) had an STD outside 1 SD of the mean. That participant was also classified as an aspirator by discriminant analysis.

For discriminant analysis, aspirators were significantly different from both the nonaspirators and the normal subjects $(p<0.01)$ (see function 1 in Fig. 2). However, the nonaspirating stroke patients and the normal subjects were not significantly different from each other $(p=0.48)$ (see function 2 in Fig. 2).

The close association of STD values for nonaspirating stroke patients and normal participants versus aspirators in this discriminant analysis is depicted in Figure 2. In function 1 the aspirators were distinctly separated from the other two groups. However, in function 2 the nonaspirators and the normal subjects were not separated from each other, factoring the results of mean, SD, and range of STD.

Table 3 provides the predicted group membership based on STD for each of the three groups. Overall, $71.2 \%$ of the 66 subjects were correctly classified. For the aspirators, 15 of 20 subjects (75\%) were correctly classified as aspirators, whereas 5 of 20 subjects $(25 \%)$ were classified as nonaspirators. None of aspirators was classified as a normal subject. For the nonaspirators, 18 of 31 subjects (58.1\%) were correctly classified, whereas 12 subjects (38.7\%) were classified as normal subjects and 1 subject (3.2\%) were classified as an aspirator. For the normal subjects, 14 of 15 subjects $(93.3 \%)$ were classified correctly, whereas only 1 subject $(6.7 \%)$ was classified as 
Table 2. The mean duration (in seconds) and standard deviations of the three groups on the STD for 5-ml and 10-ml thin liquids

\begin{tabular}{llll}
\hline Groups & & std5 & std10 \\
\hline \multirow{2}{*}{ Aspirators } & Mean & 1.28 & 1.12 \\
& SD & 0.73 & 0.53 \\
\multirow{3}{*}{ Nonaspirators } & Range & 3.03 & 1.86 \\
& Mean & 0.40 & 0.36 \\
& SD & 0.25 & 0.20 \\
Normals & Range & 0.83 & 0.86 \\
& Mean & 0.19 & 0.23 \\
& SD & 0.07 & 0.07 \\
& Range & 0.26 & 0.24 \\
\hline
\end{tabular}

$\mathrm{SD}=$ standard deviation

a nonaspirator (stroke patient). None of the normal subjects were classified as aspirator. Thus, the aspirators and the normal subjects were correctly classified more than $80 \%$ of the time, whereas nonaspirators were correctly classified only $54.8 \%$ of the time. The triggering of pharyngeal swallowing for aspirators was different than that for both the nonaspirators and the normal subjects, whereas the triggering of the pharyngeal swallow was much more similar for nonaspirating stroke patients and normals.

\section{Discussion}

In the past two decades research has continued to refine the diagnostic tools used to assess swallowing function. While we have clearly established that aspiration can cause negative health outcomes such as pneumonia, dehydration, weight loss, malnutrition, and even death $[8,9]$, we have also come to understand that the presence or absence of aspiration during a brief swallowing evaluation conducted under carefully controlled circumstances may not be sufficient to rule out that aspiration may occur in a more natural environment [10]. One of the primary physiologic causes of aspiration is a delayed onset of the pharyngeal swallow. One of the measures created to assess this aspect of swallowing is STD.

Our results indicate that STD correctly predicted the presence of aspiration $75 \%$ of the time and correctly predicted the absence of aspiration in stroke patients over $93 \%$ of the time. All aspiration occurred either before or during the swallow of thin liquids. There are two obvious implications for these results. First, we have provided data to support the clinical concept that a prolonged transition between the end of the oral stage and the beginning of the pharyngeal stage of swallow places an individual at risk for

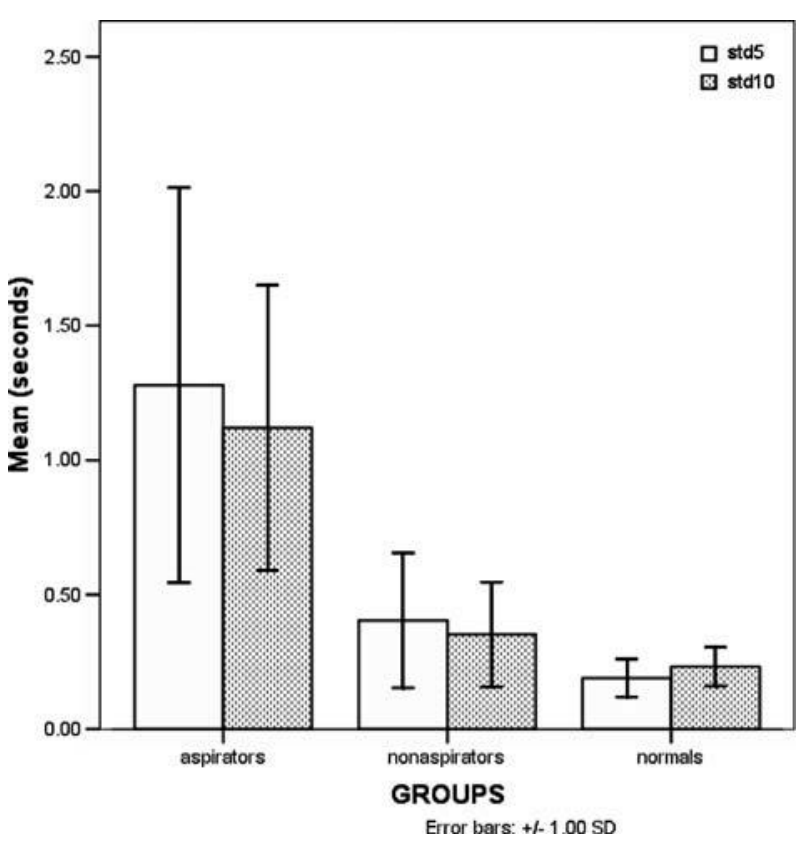

Fig. 1. Means and 1 standard deviation of STD (seconds) for 5-ml and $10-\mathrm{ml}$ thin liquids.

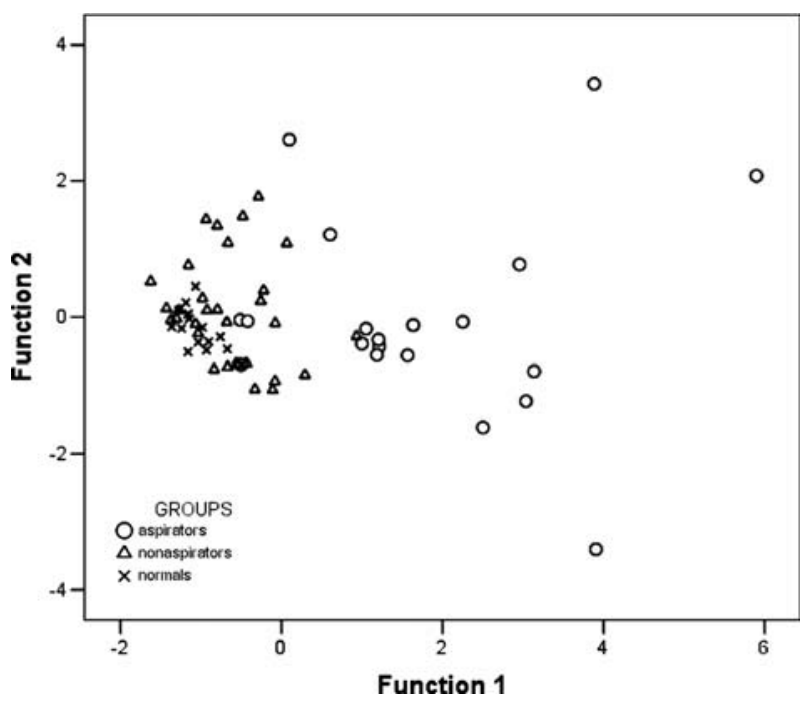

Fig. 2. The discriminant functions of three groups on the STD.

aspiration before and/or during the swallow. Second, we have provided some data (although preliminary and with a small sample) that may help us determine when STD is too long in patients who have suffered a stroke. As previously suggested, a person may not aspirate when provided a verbal prompt in an upright position. Still, if the STD is prolonged, this might indicate the need for further testing or close followup, even in the event that no aspiration is observed. Based on these data, we can roughly estimate that up to 0.5 -s STD and even 0.75 -s STD may be completely 
Table 3. The predicted group membership for the three groups on the STD

\begin{tabular}{|c|c|c|c|c|c|c|}
\hline \multicolumn{7}{|c|}{ Classification Results $^{\mathrm{a}}$} \\
\hline & & \multirow[b]{2}{*}{ Groups } & \multicolumn{4}{|c|}{ Predicted Group Membership } \\
\hline & & & Aspirators & nonaspirators & normals & total \\
\hline \multirow{6}{*}{ Original } & Count & aspirators & 15 & 5 & 0 & 20 \\
\hline & & Nonaspirators & 1 & 18 & 12 & 31 \\
\hline & & Normals & 0 & 1 & 14 & 15 \\
\hline & $\%$ & aspirators & 75.0 & 25.0 & .0 & 100.0 \\
\hline & & nonaspirators & 3.2 & 58.1 & 38.7 & 100.0 \\
\hline & & normals & .0 & 6.7 & 93.3 & 100.0 \\
\hline
\end{tabular}

a. $71.2 \%$ of original grouped cases correctly classified.

safe. On the other hand, STDs approaching 0.9 or 1.0 $\mathrm{s}$ may be considerably more dangerous. Again, this is a small sample with some overlap of numbers and the results in no way indicate a standard duration to use at this time. With such a small sample these results can be used only to alert clinicians to the potential of aspiration occurring inside and outside the fluoro suite when STDs are prolonged.

Among five misclassified aspirators, four aspirated thin liquids during the swallow. These subjects initiated hyoid excursion around $0.5 \mathrm{~s}$ after the bolus passed the ramus of mandible. Their STD is similar to nonaspirators'. The investigators noticed that these aspirators showed poor laryngeal protection during the swallow (i.e., reduced hyolaryngeal elevation and poor epiglottic tilt/seal). The bolus entered the laryngeal vestibule despite a relatively brief STD. Thus, while STD appears to be an important factor for aspiration before and during the swallow, there are other factors that can influence the results, especially for aspiration that occurs during the swallow. More research is needed with larger sample sizes to draw any more firm conclusions. It is clear, however, that STD will have to be examined in conjunction with other aspects of swallowing, such as hyolaryngeal elevation and overall laryngeal closure. It is possible that pharyngeal muscular strength might also make a difference. Crary and Baldwin [11] reported that aspirators had higher and more variable amplitude and shorter-duration hyolaryngeal movement with poor coordination of swallowing during surface electromyography (SEMG) traces. Less muscular strength may create shorter durations of hyolaryngeal excursion and, thus, poor laryngeal protection in relationship to bolus flow. Range of hyolaryngeal movement was not examined in this investigation and any connection would be purely speculative. This is to suggest only concepts that may be targeted in future research to help explain aspi- ration during the swallow when STDs are relatively normal.

One nonaspirator was classified as an aspirator in our sample. In the world of false positives, this is not a very discouraging number. Regardless, this is an individual who may have a slightly longer STD than the normal range and still not aspirate. Whether this individual may be at more risk for aspiration than others is unknown. The larger number of incorrect classifications was for nonaspirators classified as normal. This seems inordinately reasonable, however, because many stroke patients have normal swallowing function. However, it is beneficial to have data that clarify that STDs, when approaching values observed in nonstroke patients, tend not to lead to aspiration. Nonaspirating stroke patients had a slightly slower triggering of pharyngeal swallowing than normal subjects, in general, but this slower triggering was apparently not enough to make them aspirate. Again, these data provide important information regarding how much of a swallowing delay (how prolonged an STD) must be present to create an aspiration risk. Does this mean that everyone with an average STD of $1.28 \mathrm{~s}$ on $5 \mathrm{ml}$ or $1.12 \mathrm{~s}$ on $10 \mathrm{ml}$ requires treatment for a delayed swallow? Probably not. Nonetheless, future studies may zero in on a set of numbers to guide treatment in such a fashion.

While research into treatments for reducing the transition delay between the oral and the pharyngeal stages have provided little and conflicting support [12,13], new treatments are always emerging. In addition, compensatory strategies such as the chintuck procedure (touching the chin to the neck) have been demonstrated to reduce aspiration in some patients [14]. Certainly more research into both compensating for and rehabilitating delayed swallowing function is needed. What the current data provide are (a) additional evidence that there is a strong relationship between delayed swallowing function and 
aspiration, (b) evidence to support the definition of STD as an accurate depiction of this swallowing delay that helps to distinguish normal from potentially dangerous swallowing transitions, and (c) some numerical values that may prove valuable for distinguishing aspiration risk based on stage transition. These values, of course, will need much more data to support or refute their validity but may prove helpful in time for providing guidelines regarding when prolonged means too long for oral-pharyngeal stage transition.

This study has several limitations such as a small number of subjects, VFSE data derived from a previous investigation, and a limited number of normal subjects who fell into the range of the stroke patients' ages rather than point-to-point age and gender matching. Future study should focus on timing of aspiration and STD as a contributing factor with more subjects. In addition, more detailed physiologic measurements like EMG may help understand the temporal characteristics of triggering the pharyngeal swallow for patients poststroke.

Acknowledgments. This work was made possible in part by a grant from the Department of Veterans Affairs Office of Academic Affiliations and a grant from the Department of Veterans Affairs.

\section{References}

1. Logemann JA: Evaluation and Treatment of Swallowing Disorders. Austin, TX: Pro-Ed, 1998

2. Cook IJ, Dodds WJ, Dantas RO, Kern MK, Massey BT, Shaker R, Hogan WJ: Timing of videofluoroscopic, manometric events, and bolus transit timing during the oral and pharyngeal phases of swallowing. Dysphagia 4:8-15, 1989
3. Robbins JA, Hamilton JW, Lof GL, Kempster GB: Oropharyngeal swallowing in normal adults of different ages. Gastroenterology 103:823-829, 1992

4. Robbins JA, Levine RL: Swallowing after unilateral stroke of the cerebral cortex: preliminary experience. Dysphagia 3:11-17, 1998

5. Kim Y, McCullough GH, Asp CW: Temporal measurements of pharyngeal swallowing in normal populations. Dysphagia 20:290-296, 2005

6. McCullough GH, Wertz RT, Rosenbek JC: Sensitivity and specificity of clinical/bedside examination signs for detecting aspiration in adults subsequent to stroke.J Commun Disord 34:55-72, 2001

7. McCullough GH, Wertz RT, Rosenbek JC: Age, Gender, Size, Consistency Effects on Swallowing Function in Adults Between 21 and 99 Years of Age. Albuquerque, NM: Presented at the 10th annual meeting of Dysphagia Research Society, 2001

8. Langmore SE, Terpenning MS, Schork A, Chen Y, Murray JT, Lopatin D, Loesche WJ: Predictors of aspiration pneumonia: how important is dysphagia? Dysphagia 13(2):69-81, 1998

9. Holas MA, DePippo KL, Reding MJ: Aspiration and relative risk of medical complications following stroke. Arch Neurol 51(10):1051-1053,1994

10. Groher ME: The detection of aspiration and videofluoroscopy. Dysphagia 9:147-148, 1994

11. Crary MA, Baldwin BO: Surface electromyographic characteristics of swallowing in dysphagia secondary to brainstem stroke. Dysphagia 12(4):180-187, 1997

12. Bisch EM, Logemann JA, Rademaker AW, Kahrilas PJ, Lazarus CL: Pharyngeal effects of bolus volume, viscosity, and temperature in patients with dysphagia resulting from neurologic impairment and in normal subjects. J Speech Hear Res 37(5):1041-1059, 1994

13. Rosenbek JC, Robbins J, Fishback B, Levine RL: Effects of thermal application on dysphagia after stroke.JSpeech Hear Res 34(6):1257-1268, 1991

14. Welch MV, Logemann JA, Rademaker AW, Kahrilas PJ: Changes in pharyngeal dimensions effected by chin tuck. Arch Phys Med Rehabil 74(2):178-181, 1993 